Z. klin. Chem. u. klin. Biochem.

8. Jg., S. $145-148$, März 1970

\title{
Die Herkunft der Fruchtwasser-Enzyme
}

\author{
Von H. GeYer \\ Aus der Universitäts-Frauenklinik Freiburg i. Breisgau (Direktor: Prof. Dr. H. Wimböfer)
}

(Eingegangen am 17. November 1969)

Die spezifischen Aktivitäten von Lactat- und Malatdehydrogenase, Lcucinaminopeptidase, Alkalischer Phosphatase, Saurer Phosphatase, Aspartat- und Alanintransaminase, Glucose-6-phosphatdehydrogensase, $\alpha$-Amylase und Cholinesterase in Fruchtwasser wurden gemessen und mit den entsprechenden Enzymmustern von Eihaut, Placenta, Serum aus Nabelvenenblut, Neugeborenen-Urin, NeugeborenenSpeichel, Mekonium und Vaginal-Schleim verglichen - ebenso die Isoenzym-Muster der Lactat- und Malatdehydrogenase von Fruchtwasser mit denen von Eihaut, Placenta und Serum aus Nabelvenenblut. Aufgrund dieser Vergleiche wurde gefolgert, daß die Fruchtwasser-Enzyme im wesentlichen aus der Eibaut stammen und durch Autolyse von Eihautzellen ins Fruchtwasser gelangen.

\section{The origin of the encymes of the amniotic fluid}

The specific activities of lactate and malate dehydrogenase, leucine aminopeptidase, alkaline phosphatase, acid phosphatase, aspartate and alanine transaminase, glucose- 6 -phosphate dehydrogenase, $\alpha$-amylase and cholinesterase in amniotic fluid were measured. They were then compared with the corresponding enzyme patterns in amnion, placenta, cord blood serum, neonatal urine and saliva, meconium and vaginal mucus. Furthermore the isoenzyme patterns of lactate and malate dehydrogenase in amniotic fluid were compared with those of amnion, placenta and cord blood serum. On the basis of these comparisons it was concluded that amniotic fluid enzymes originate principally in the amnion and enter the amniotic fluid by autolysis of the amniotic cells.

Im menschlichen Fruchtwasser sind Enzyme enthalten, deren Aktivität etwa so hoch liegt wie in menschlichem Serum (1). Als Quelle für diese Enzyme kommen in Frage: fetaler Urin, fetaler Speichel, der Magen-DarmTrakt und die Respirationsorgane des Feten, ferner fetales und maternales Serum, Placenta und Eihaut und schließlich noch Verunreinigungen durch Vaginalschleim und Blut.

Wir versuchten, ihre Herkunft an Hand von EnzymMustern zu klären. Dabei kamen wir zu dem Ergebnis, daß die Fruchtwasser-Enzyme im wesentlichen aus der Eihaut stammen und daß der Anteil anderer Quellen demgegenüber gering sein muß.

\section{Material und Methoden}

Die Fruchtwasserproben wurden bei Blasensprengungen gewonnen. Bluthaltige Proben wurden verworfen. Vernixflocken ließen sich durch Filtrieren und Zentrifugieren entfernen. Amnionproben und zerschnittenes Placentagewebe wurden mit physiologischer $\mathrm{NaCl}-\mathrm{Lösung}$ blutfrei gewaschen und mit dem dreifachen Volumen $0,066 \mathrm{M}$ Phosphatpuffer $\mathrm{pH} 7,4$, der 0,04\% Triton-X-100 enthielt, im Ultraturrax-Homogenisator 2 Min. unter Eiskühlung homogenisiert. Nach $10 \mathrm{Min}$. Zentrifugieren bei $30000 \mathrm{~g}$ wurde der Uberstand für die Messungen verwendet, wobei er gegebenenfalls mit Phosphatpuffer weiterverdünnt werden mußte.

Die Messung der Aktivitäten von Lactatdehydrogenase (EC 1.1.1.27), Malatdehydrogenase (EC 1.1.1.37), Leucinaminopeptidase (= Aminosäure-Arylamidase) (EC 3.4.1.1), Alkalischer Phosphatase (EC 3.1.3.1), Saurer Phosphatase (EC 3.1.3.2), Aspartattransaminase (EC 2.6.1.1), Alaninttansaminase (EC 2.6.1.2), Glucose-6-phosphatdehydrogenase (EC 1.1.1.49), $\alpha$-Amylase (EC 3.2.1.1) und Cholinesterase (EC 3.1.1.8) ist einer in früheren Arbeit beschrieben (1). Die elektrophoretische Trennung der Lactatdehydrogenase in die Isoenzyme LDH-I bis LDH-V und der Malatdehydrogenase in die Isoenzyme MDH-I und MDH-II wurde ebenfalls in früheren Arbeiten beschrieben $(2,3)$.

\section{Ergebnisse}

In einer friiberen Untersuchung (1) hatten wir für 12 normale Fruchtwasserproben Enzymaktivitäten gefunden, deren Mittelwert und Standardabweichung in Tabelle 1 angegeben sind. In der gleichen Tabelle sind die Mittelwerte zweier Fruchtwasserproben von einer Patientin (F. A.), bei der nach einem Blasensprung Fruchtwasser

Tab. 1

Enzymaktivitäten ( $\mathrm{mU} / \mathrm{ml}$ ) im normalen Fruchtwasser: a) Mittelwerte $\bar{x}$ und Standardabweichung s aus 12 untersuchten Proben (1). b) Enzymaktivitäten (mU/ml) im Fruchtwasser einer Patientin ( $F$. A.) mit vorzeitigem Blasensprung

Abkürunen. $\mathrm{DH}=$ aktat- $\mathrm{MDH}=$ Malatdehydrogenase LAP $=$ Leucinaminopeptidase; $A P=$ Alkalische, SP $=$ Saure Phosphatase GOT $=$ Aspartat-, GPT = Alanintransaminase; G-6-PDH = Glucose-6-phosphatdehydrogenase; ChE = Cholinesterase

\begin{tabular}{|c|c|c|c|c|c|c|c|c|c|c|}
\hline & LDH & MDH & LAP & AP & SP & GOT & GPT & G-6-PDH & $\alpha$-Amylase & ChE \\
\hline $\begin{array}{l}\text { a) Mittelwert }(n=12) \bar{x} \\
\pm s\end{array}$ & $\begin{array}{l}238,9 \\
108,6\end{array}$ & $\begin{array}{l}82,4 \\
28,8\end{array}$ & $\begin{array}{l}2,5 \\
1,8\end{array}$ & $\begin{array}{l}27,2 \\
11,9\end{array}$ & $\begin{array}{l}1,35 \\
1,03\end{array}$ & $\begin{array}{l}8,6 \\
3,8\end{array}$ & $\begin{array}{l}1,6 \\
0,9\end{array}$ & $\begin{array}{l}0,8 \\
0,9\end{array}$ & $\begin{array}{l}56,0 \\
49,1\end{array}$ & $\begin{array}{l}32,8 \\
45,8\end{array}$ \\
\hline b) Pat. F. A. (2 Proben) & 319,6 & 110,4 & 4,0 & 6,6 & 0,0 & 12,0 & 1,8 & 0,0 & 110,0 & 0,0 \\
\hline
\end{tabular}


über längere Zeit lief, verzeichnet. Zur besseren Übersicht sind in Abbildung 1 diese Enzymaktivitäten in der Weise wiedergegeben, daß die LDH-Aktivität $=100$ gesetzt wurde. Damit ergaben sich gut vergleichbare relative

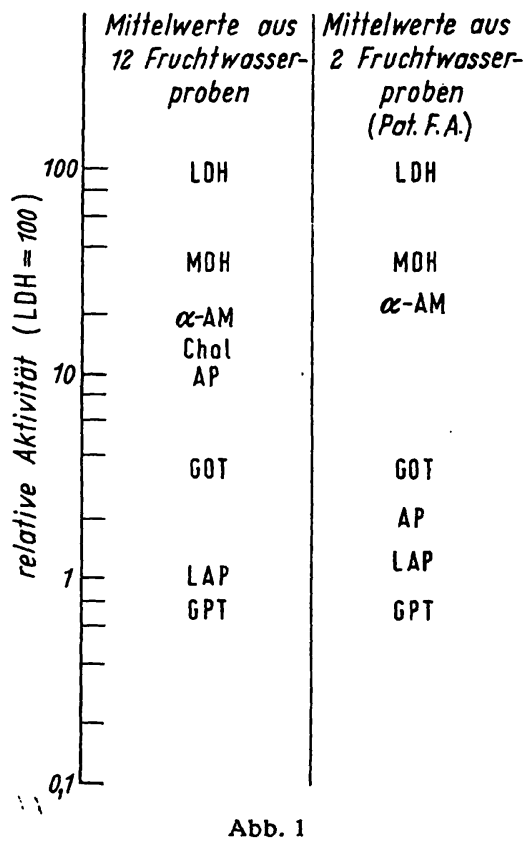

Relative Enzymaktivitäten (Lactatdehydrogenase-Aktivität $=100$ ) in 12 normalen Fruchtwasserproben und im Fruchtwasser einer Patientin 12 normalen Fruchtwasserproben und im Fruchtwasser einer Patientin
(F. A.) mit vorzeitigem Blasensprung. Abkürzungen vgl. Tab. 1

Aktivitäten. Die relativen Aktivitäten im Fruchtwasser der Pat. F. A. stimmten mit denen in normalen Fruchtwasserproben überein. Eine Ausnahme machten nur Cholinesterase und alkalische Phosphatase.

Abbildung 2 zeigt die Isoenzym-Verteilung von Lactatdehydrogenase in Fruchtwasser. Die hier wiedergegebenen Werte sind Mittelwerte von zwei verschiedenen

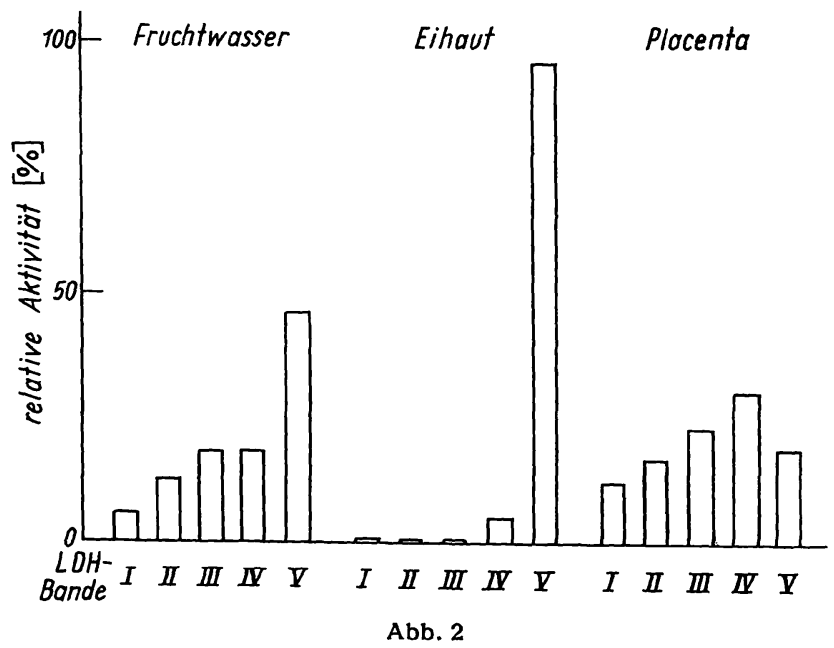
Lactatdehydrogenase-Isoenzym-Muster in Fruchtwasser, Eihaut und

Fruchtwasserproben, deren Lactatdehydrogenase-Aktivität sehr hoch lag $(400 \mathrm{mU} / \mathrm{m} /)$. Bei Fruchtwasserproben mit durchschnittlicher Lactatdehydrogenase-Aktivität (um $240 \mathrm{mU} / \mathrm{ml}$ ) war keine exakte Messung der Iso-
enzym-Verteilung möglich, sondern nur eine Auswertung durch Färbung der Elektrophoresestreifen. Dabei war nahezu alle Aktivität in LDH-V, während LDH-IV und LDH-III nur Spuren enthielten. LDH-II und LDH-I waren überhaupt nicht vorhanden.

Weiterhin sind zum Vergleich die LactatdehydrogenaseIsoenzym-Muster von Eihaut und Placenta wiedergegeben. Auch hier handelt es sịch um Mittelwerte von jeweils 2 Proben. Eihaut enthielt im wesentlichen LDH-V. Die Verteilung glich auffallend derjenigen bei Fruchtwasserproben mit durchschnittlicher Lactatdehydrogenase-Aktivität. Dagegen fanden wir in Placentagewebe eine gleichmäßige Aktivitätsverteilung mit dem Maximum bei LDH-IV.

Die Malatdehydrogenase-Isoenzym-Muster von Fruchtwasser, Eihaut, Placenta und Serum aus Nabelvenenblut (in dieser Arbeit kurz „fetales Serum" genannt) sind in Abbildung 3 wiedergegeben. Bei Fruchtwasser war die

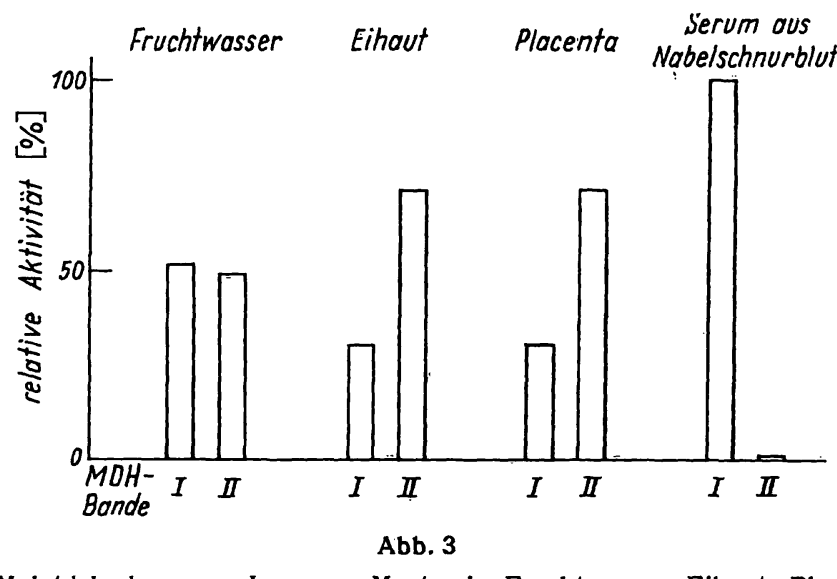

Malatdehydrogenase-Isoenzym-Muster in Fruchtwasser, Eihaut, Placenta und Serum aus Nabelschnurblut

Aktivität zu gleichen Teilen auf MDH-I und MDH-II verteilt. Eihaut und Placenta hatten jeweils $1 / 3$ der Aktivität in MDH-I. Im fetalen Serum war nur MDH-I vorhanden; MDH-II fehlte völlig.

Die in Abbildung 4 und 5 wiedergegebenen relativen Enzymaktivitäten von Fruchtwasser, Eihaut, Placẹnta, Mekonium, fetalem Serum, Urin und Speichel von Neugeborenen und Vaginalschleim ermöglicht einen Vergleich der Enzymmuster. Typische Zellenzyme, wie Lactat- und Malatdehydrogenase, Aspartat- und Alanintransaminase, zeigten bei Fruchtwasser und Eihaut sehr ähnliche Verteilungsmuster. Die übrigen untersuchten Flüssigkeiten und Placentagewebe hatten dagegen abweichende Enzym-Muster, wobei hohe Gehalte an alkalischer Phosphatase, $\alpha$-Amylase, Leucinaminopeptidase und Cholinesterase hervorzuheben sind.

Die spezifische Aktivität der Lactatdehydrogenase ist in $\mathrm{mU} / \mathrm{m} l$ oder $\mathrm{mU} / \mathrm{g}$ Feuchtgewicht für die jeweils untersuchten Körperflüssigkeiten und Gewebe in den $\mathrm{Ab}$ bildungen angegeben. In Vernix war keines der untersuchten Enzyme feststellbar. 


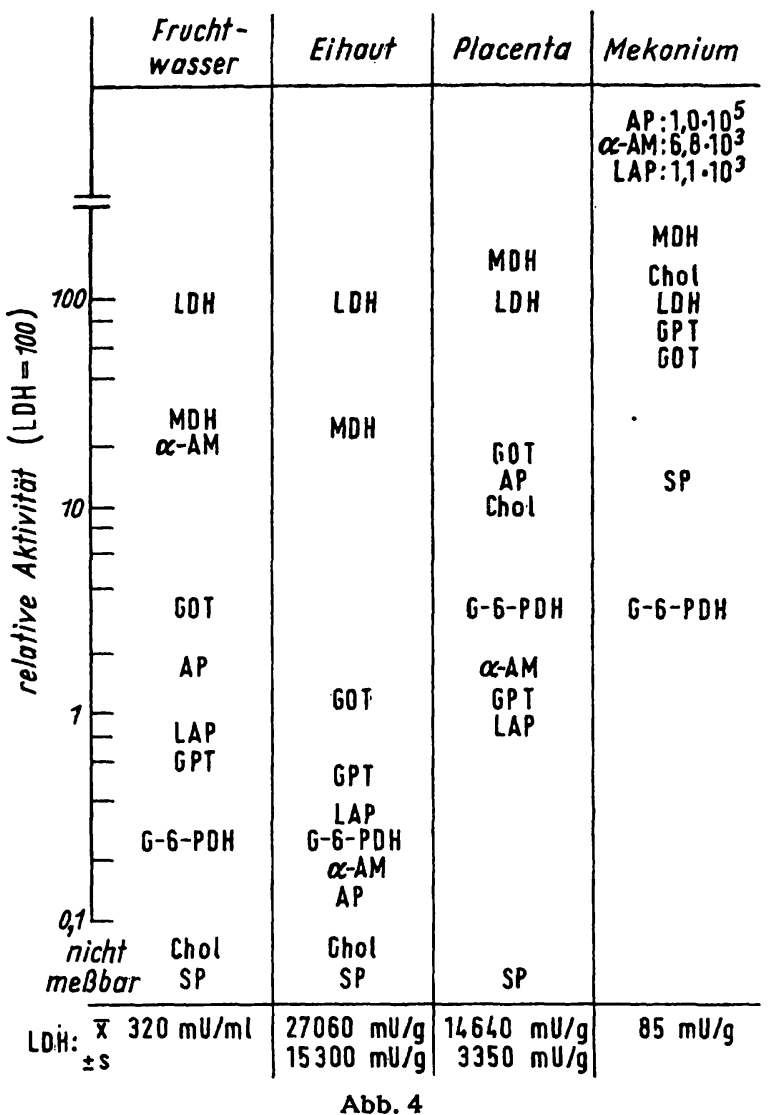

Relative Enzymaktivitäten (Lactatdehydrogenase-Aktivität $=100$ ) in Fruchtwasser, Eihaut, Placenta und Mekonium. (Angabe der Lactatdehydrogenase-Aktivität in $\mathrm{mU} / \mathrm{ml}$ bzw. $\mathrm{mU} / \mathrm{g}$.) Abkürzungen vgl.

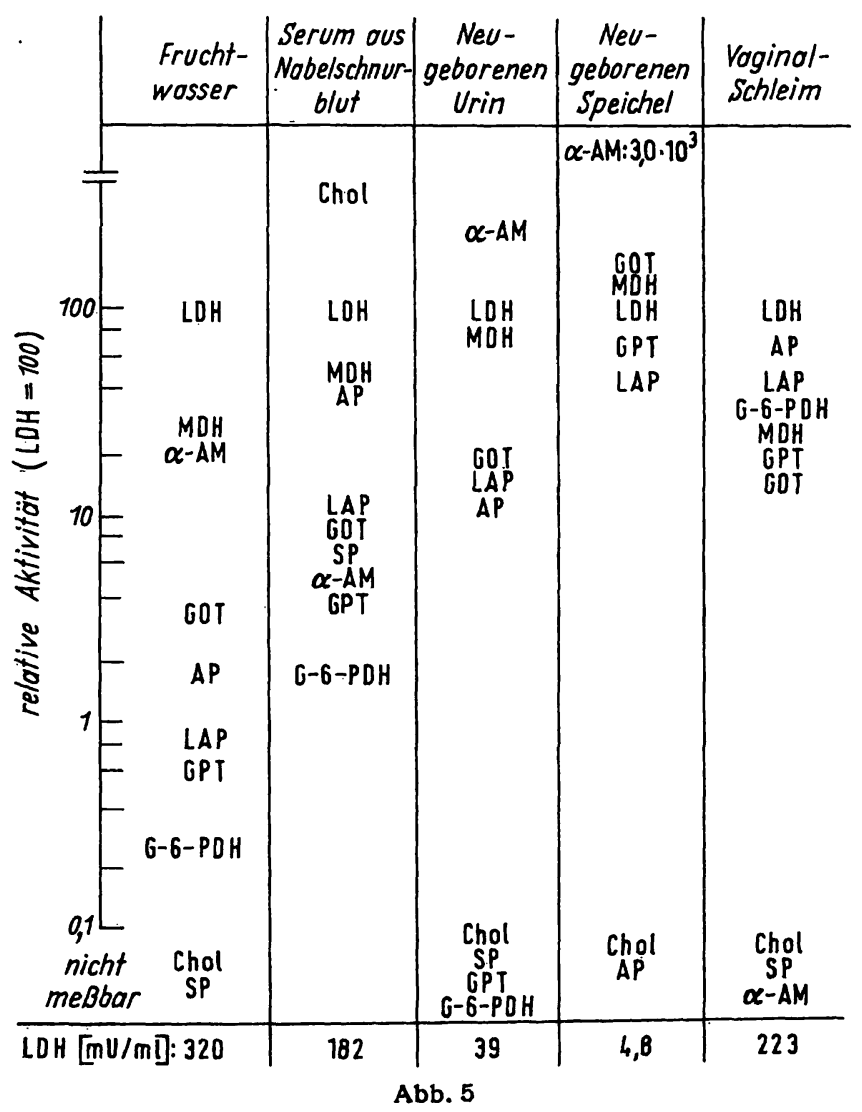

Relative Enzymaktivitäten (Lactatdehydrogenase-Aktivität $\overline{=} 100$ ) in Fruchtwasser, Serum aus Nabelschnurblut, Neugeborenen-Urin und -Speichel und Vaginalschleim. (Angabe der Lactatdehydr.

\section{Diskussion}

Berechnet man aus den spezifischen Aktivitäten der Tabelle 1 die relativen Aktivitäten mit Lactatdehydrogenase $=100$ als Bezug, dann findet man Übereinstimmung $z$ wischen den relativen Aktivitäten von normalem Fruchtwasser und von den Fruchtwasserproben der Patientin F. A. Nur Cholinesterase, die bei Patientin F. A. ganz fehlte, und Alkalische Phosphatase, die an der unteren Grenze der Erfaßbarkeit war, bilden eine Ausnahme. Der Grund für diese Unterschiede dürfte sein, daß sich unter den 12 normalen Fruchtwasserproben einige befanden, die Spuren von Blut oder Mekonium enthielten. Diese Verunreinigungen brachten Aktivitäten von Cholinesterase bzw. Alkalischer Phosphatase. Bei der Patientin F. A. dagegen fehlten diese Verunreinigungen. Daher konnten wir die relativen Enzym-Aktivitäten dieses Fruchtwassers mit den relativen Enzym-Aktivitäten der untersuchten Gewebe und Flüssigkeiten vergleichen.

Der Vergleich zeigte eine Ähnlichkeit zwischen den Enzymmustern von Fruchtwasser und Eihaut (Abb. 4). Von zehn untersuchten Enzymen stimmten acht jeweils überein. Eine wesentliche Abweichung zeigte nur $\alpha$ Amylase, die in Eihaut fast nicht vorhanden war. Der Quotient Lactat-: Malatdehydrogenase betrug bei Fruchtwasser und Eihaut 3,0, bei Placentagewebe dagegen 0,5. Neugeborenen-Urin (Abb. 5) hatte nicht nur ein anderes Enzymmuster, sondern schied auch auf Grund seiner niederen Aktivitäten als wesentliche Enzymquelle aus. Das gleiche gilt von Speichel Neugeborener und von Vaginalschleim. Mekonium kommt wegen der sehr hohen Gehalte an alkalischer Phosphatase, $\alpha$-Amylase, Leucinaminopeptidase und Cholinesterase nicht in Frage. Aus dem fetalen Serum konnten die Fruchtwasser-Enzyme nicht herrühren, denn dann müßte Cholinesterase im Fruchtwasser hoch sein.

Eine weitere Sicherheit brachte die Untersuchung der Isoenzyme (Abb. 2). In Fruchtwasser bestand die Lactatdehydrogenase hauptsächlich aus LDH-V. Namentlich in Fruchtwasser mit normaler Lactatdehydrogenase-Aktivität war fast nur LDH-V zu beobachten. Ebenso enthielt Eihaut fast nur LDH-V. Placentagewebe zeigte eine andere Verteilung mit dem Aktivitätsmaximum in LDH-IV.

Da fetales Serum nur MDH-I enthielt (Abb. 3), konnte die Malatdehydrogenase des Fruchtwassers, die aus $\mathrm{MDH}-\mathrm{I}$ und MDH-II bestand, nicht aus dem kindlichen Serum kommen. Für Alanintransaminase konnten WORASCHK und RöpKE (4) im Tierversuch nachweisen, $\mathrm{daß}$ das Enzym nicht vom mütterlichen Serum ins Fruchtwasser gelangt.

Unsere Untersuchungen zeigen, daß die Enzyme des Fruchtwassers im wesentlichen aus der Eihaut stammen. Selbstverständlich gilt das nicht ausschließlich, doch muß der Beitrag, den etwa der fetale Urin oder der fetale Speichel liefert, sehr klein sein. Ein Durchtritt von Enzymen der Placenta oder des kindlichen Serums durch die Eihaut ist offenbar nicht möglich. Das be- 
stätigt die Vermutung Rrmbachs (5), der annimmt, die Placentaschranke intakter Placenten sei nur für Substanzen mit Molekulargewichten $<1000$ durchlässig. Auf Grund unserer Untersuchungen an infarzierten Placenten (1) können wir diese Aussage dahingehend erweitern, daß die Eihaut selbst bei stark infarzierten Placenten mit ausgedehnten Nekroseherden für Enzymmoleküle undurchlässig ist.

Weiterhin folgern wir aus unseren Ergebnissen, daß die Eihaut nicht zur Sekretion der von uns untersuchten Enzyme fähig ist. Vielmehr dürften diese Enzyme mit dem die Eihäute durchdringenden Wasserstrom durch Autolyse von Eihautzellen in das Fruchtwasser gelangen. Das entspräche den Vorstellungen Hubers, wonach Fruchtwasser kein Sekret des Amnionepithels ist
(6). Unsere Untersuchungen zeigen nur, daß Eihaut keine Enzyme sezerniert. Das hat nichts mit dem aktiven Transport zu tun, wie er etwa von JANTZEN (7) für niedermolekulare Substanzen angenommen wird, oder mit dem DonNAN-Gleichgewicht, von dem SCHREINER (8) spricht.

Da $\alpha$-Amylase nicht regelmäßig in Fruchtwasser zu finden ist, muß für dieses Enzym angenommen werden, $\mathrm{da} ß$ es durch eine andere Quelle'als Eihaut - etwa aus dem Magen-Darm=Trakt - in stark wechselnden Mengen ins Fruchtwasser gelangt. Würde es von der Eihaut sezerniert, müßte es stets im Fruchtwasser zu messen sein.

Fräulein E. Buchwald und Fräulein U. Nolte sei für ihre ausgezeichnete technische Mitarbeit gedankt.

\section{Literatur}

1. Geyer, H. und I. Schneider, diese Z. 8, 141 (1970). 2. GeYer, H., Klin. Wschr. 46, 443 (1968). - 3. GeYer, H., Zschr. analyt. Chem. 243, 578 (1968). - 4. WoraschK, H. J. und H. Röpke, Zschr. Geburtsh., Stuttgart 170, 193 (1969). - 5. RıM-
BACH, E., Arch. Gynäk. 198, 198 (1963). - 6. HubER, R. und D. KöHNE, Gynäkologia 155, 160 (1963). - 7. JANTzEN, K., Geburtsh. u. Frauenhk. 27, 1102 (1967). - 8. SchreINER, W. E., „Fruchtwasser und Fetus“. Bibl. gynaec. Fasc. 31 (1964).
Dr. H. Geyer 78 Freiburg i. Br. Hugstetterstr. 55 


\section{Analytica70}

Internationale Fachausstellung

\section{für Biochemische Analyse}

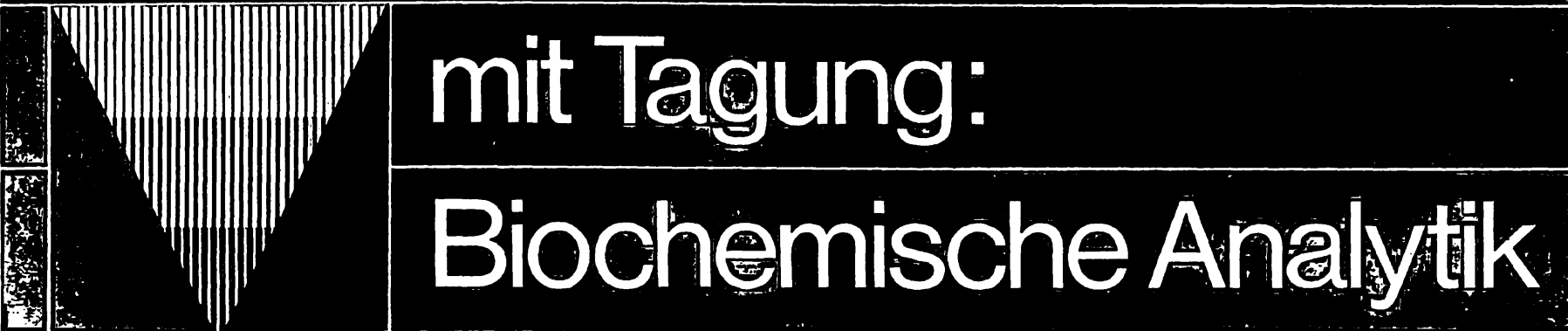

\section{München 29. April-2. Mai

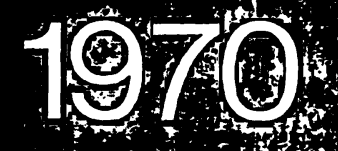

Auskunft: Münchener Messe- und Ausstellungsgesellschaft mbH · 8000 München 12,Theresienhöhe 13 · Postfach 200 · Telefon (0811) 76711·Telex:05-212086 ·TA:AMEC

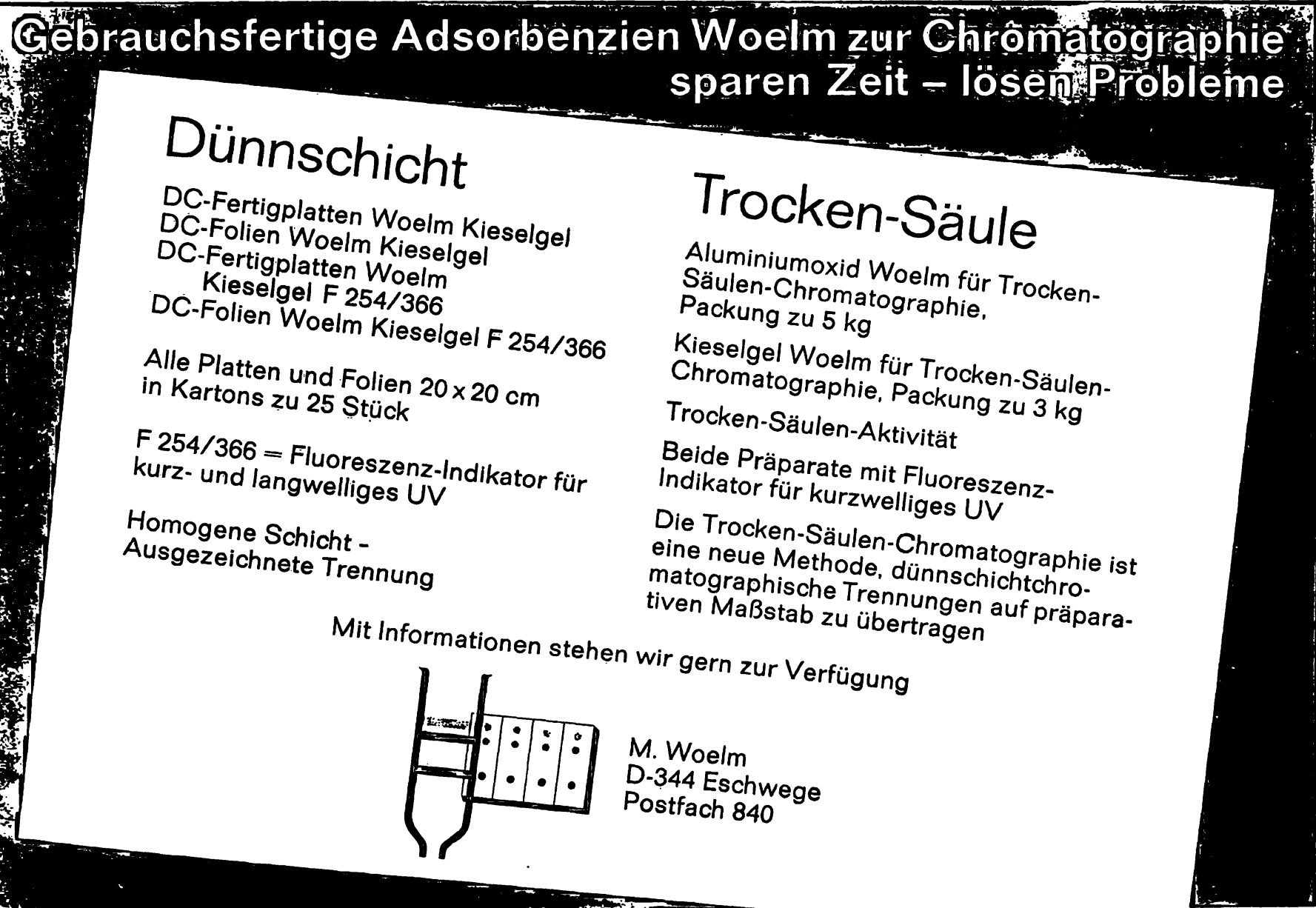




\title{
Buddecke \\ Grundriß der Biochemie,
}

\author{
für Studierende der Medizin, Zahnmedizin und Naturwissenschaften
}

\author{
Von Prof. Dr. E. Buddecke \\ Mit mehr als 400 Formeln, Tabellen und Diagrammen \\ XXXII, 499 Seiten. 1970. Plastik flexibel DM 27,50
}

\begin{abstract}
Die progressive Zunahme des biochemischen Fachwissens erfordert eine überschaubare und zusammenfassende Darstellung der Biochemie als Hilfsmittel für den Unterricht. Der Grundriß der Biochemie von E. Buddecke gliedert den Wissensstoff in die Kapitel „Stoffe und Stoffwechsel“, „Stoffwechselregulation“ und „Funktionelle Biochemie der Organe und Gewebe“ mit dem Ziel, durch knappe Darstellung gesicherter Fakten und gezielte Stoffauswahil'dem Leser einerseits eine rasche Information zu bieten, andererseits jedoch auf die vielfăltigen Beziehungen und Anwendungsmöglichkeiten der Biochemie zur klinischen Chemie und Molekularpathologie hinzuweisen, um damit dem Studierenden der Medizin und Zahnmedizin sowie der anderen Gebiete der Naturwissenschaften das Verständnis klinischen Fachwissens zu erleichtern.
\end{abstract}

\section{Inhaltsübersicht}

Stoffe und Stoffwechsel

Chemische Zusammensetzung und Stoffwechsel der Zelle - Der Stoffwechsel als chemische Reaktionsfolge Enzyme - Coenzyme - Aminosäuren - Nucleinsäuren Proteine - Glucose und Kohlenhydrate - Lipide - Citratzyklus und biologische Oxydation - Porphyrine - Wasserhaushalt - Mineralhaushalt

Stoffwechselregulation

Selbstregulation durch Rückkopplung - Regulation durch Metabolitkonzentrationen - Enzymkonkurrenz Hormone
Einführung - Glanduläre Hormone - Gewebshormone

Vitamine

Definition und Klassifizierung - Vitamine mit Coenzymfunktion - Vitamine ohne Coenzymfunktion - Vitaminähnliche Wirkstoffe

Funktionelle Biochemie der Organe und Gewebe

Biochemie der Zelle - Blut - Leber - Verdauung und Resorption - Niere und Urin - Muskel - Nervengewebe Binde- und Stützgewebe - Wachstum und Abwehr 